\title{
Achieving large slip with superhydrophobic surfaces: Scaling laws for generic geometries
}

\author{
Christophe Ybert, ${ }^{\text {a) }}$ Catherine Barentin, Cécile Cottin-Bizonne, \\ Pierre Joseph, and Lydéric Bocquet \\ Laboratoire de Physique de la Matière Condensée et Nanostructures, UMR 5586, \\ Université Claude Bernard Lyon 1 et CNRS, 69622 Villeurbanne Cedex, France
}

(Received 16 February 2007; accepted 16 October 2007; published online 3 December 2007)

\begin{abstract}
We investigate the hydrodynamic friction properties of superhydrophobic surfaces and quantify their superlubricating potential. On such surfaces, the contact of the liquid with the solid roughness is minimal, while most of the interface is a liquid-gas one, resulting in strongly reduced friction. We obtain scaling laws for the effective slip length at the surface in terms of the generic surface characteristics (roughness length scale, depth, solid fraction of the interface, etc.). These predictions are successfully compared to numerical results in various geometries (grooves, posts or holes). This approach provides a versatile framework for the description of slip on these composite surfaces. Slip lengths up to $100 \mu \mathrm{m}$ are predicted for an optimized patterned surface. () 2007 American Institute
\end{abstract} of Physics. [DOI: 10.1063/1.2815730]

\section{INTRODUCTION}

The design and fabrication of micro- and nanopatterned nonwetting surfaces have received much attention in recent years. ${ }^{1,2}$ This was initially motivated by the peculiar staticwetting properties of such surfaces, associated with the socalled superhydrophobic effect. The natural nonwettability of the flat substrate, as quantified by the liquid contact angle, is enhanced by the underlying roughness, reaching values close to $180^{\circ}{ }^{3}$ Depending on the characteristics of the liquid-solid interface, two different classes of superhydrophobic states are exhibited, namely the so-called Wenzel and Cassie states. For the Wenzel case, ${ }^{4}$ the liquid impregnates the roughness, while for the Cassie (fakir) state, ${ }^{5}$ the liquid interface is restricted to the top of the roughness, the roughness being occupied by a gas phase. The relative stability of the two states depends on the surface structure characteristics (height and lateral scale, etc.) ${ }^{6}$ and the experimental conditions (liquid pressure, etc.). ${ }^{2,7}$ While a strong dissipation is expected in the Wenzel state as the liquid flow follows the contour of the roughness, it was predicted by Philip ${ }^{8,9}$ that a composite interface in the Cassie state should display a low frictionsuperlubricating behavior.

Such a superlubricating behavior is particularly attractive in the context of micro- and nanofluidic devices. As downsizing leads to an increased surface-to-volume ratio, superlubricating properties of textured nonwetting surfaces provide a way to bypass the huge increase in hydrodynamic resistance that comes with system miniaturization. ${ }^{10}$ Accordingly, surface effects become key factors in the understanding of the motion of liquids at ever smaller scales. A reduced wall friction is associated with a breakdown of the no-slip boundary condition of the hydrodynamic velocity field at the surface, leading to wall slippage. Slippage is described by

\footnotetext{
${ }^{a)}$ Electronic mail: Christophe.Ybert@1pmen.univ-lyon1.fr. URL: http:// lpmen.univ-lyon 1.fr/interfaces_fluides/.
}

the Navier boundary condition $(\mathrm{BC})^{11}$ for the velocity field at the surface, $b_{\text {eff }} \partial_{z} v=v_{w}$, where $b_{\text {eff }}$ is the effective slip length, $v_{w}$ is the slip velocity at the wall, and $z$ is the normal coordinate to the wall.

Since the first experiments involving rolling drops, ${ }^{12}$ a few experimental works have reported the characterization of friction properties of superhydrophobic surfaces. ${ }^{13-19}$ All experimental studies confirm large slippage on microtextured $^{13,14}$ and nanotextured ${ }^{16-19}$ nonwetting surfaces, which are orders of magnitude higher than what is achievable with liquid on smooth nonwetting walls. Typically, slip lengths in the micrometer range have been demonstrated on superhydrophobic surfaces, while it remains within the tens of nanometers range on a smooth hydrophobic surface. ${ }^{20-23}$

On the theoretical side, flows on composite interfaces (combining solid and gas areas) were first tackled theoretically by Philip ${ }^{8,9}$ and more recently by Lauga and Stone ${ }^{24}$ and Cottin-Bizonne et $a l .{ }^{25}$ Numerical approaches have also been followed, either at the molecular scale using molecular dynamics $^{6,25}$ or at larger mesoscopic scales using, e.g., Lattice-Boltzmann methods. ${ }^{26}$ At the hydrodynamic level, the composite surface is modeled as a spatially dependent boundary condition, with a no-slip BC on the solid surface and a shear-free $\mathrm{BC}$ on the liquid-vapor interface.

However, the hydrodynamic flow on a mixed $\mathrm{BC}$ is difficult to solve in practice and analytical results are only available in simple geometries. These latter essentially reduce to the case of a flow on stripes (either parallel or perpendicular to the external flow), as solved by Philip and Lauga-Stone, so that results for more complex geometries, even the rather simple case of a regular array of posts, are still lacking. In particular, for a given nanotexture (characterized by its morphology, height, length scale, etc.) investigated experimentally, ${ }^{17-19}$ there exists no prediction for the amount of slippage that is to be expected. Even simple questions such as whether slippage on an array of posts is ex- 


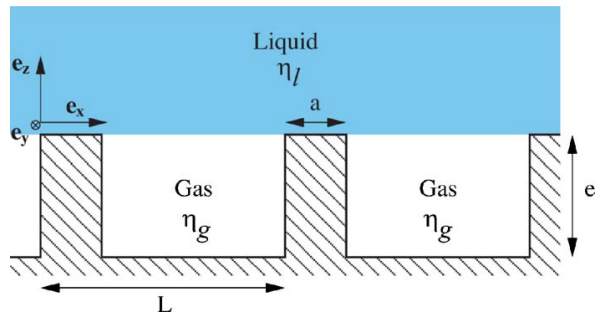

FIG. 1. (Color online) Sketch of the liquid interface at a superhydrophobic wall in the Cassie state. $L, e$ : roughness periodicity and height; $a$ : typical length scale for solid/liquid contact areas.

pected to be larger than for a stripe geometry remain without any answer up to now.

Thus, a quantitative understanding of liquid friction past superhydrophobic surfaces is still challenging. The design of optimized interfaces faces a lack of predictive tools linking the wall characteristics-texture geometry (pattern type and dimensions) and chemistry (setting the intrinsic slip length over the smooth solid) - to the final slippage properties.

In the present work, we propose analytical expressions for the slip length on superhydrophobic surfaces in the form of scaling laws in terms of the texture properties. We quantify furthermore how dissipation into the gas layer and the curvature of the liquid-gas interface may affect the final result. These predictions are successfully compared to numerical calculations of the slip lengths both by direct resolution of the hydrodynamic equations and by finite-element methods. Altogether, these results are used to discuss the merits of different generic surface geometries-stripes, pillars, holes-with respect to the resulting frictional properties. Finally, we show how these simple analytical laws can be used to link basic surface parameters to the surface slip length in order to anticipate and optimize the surface frictional properties.

\section{A SCALING LAW APPROACH FOR SLIPPAGE}

We examine in this part the problem of an idealized superhydrophobic surface in the Fakir state (sketched in Fig. 1) where a liquid slab lies on top of the surface roughness.

The liquid-gas interface is assumed to be flat (no meniscus curvature), so that the modeled superhydrophobic surface appears as a perfectly smooth surface with a pattern of BCs. The latter BCs are taken as no-slip over solid/liquid areas and shear-free over gas/liquid regions (Fig. 1). We denote as $L$ the roughness periodicity and $a$ the typical length scale of solid/liquid areas. The fraction of such solid/liquid areas will be denoted $\phi_{s}$. We assume that the fluid is described by the Stokes equation, i.e., that the Reynolds number is very small. This is a pertinent limit for microfluidic devices. Overall, the situation we investigate is similar to those studied in the simple geometries previously considered in Refs. 8, 9, 24, and 25.

In this idealized surface description, it should be recognized that two of the assumptions have a possible influence on the surface frictional properties. First, by assuming flat menisci, we have neglected an additional mechanism for mo- mentum transfer between liquid and wall. ${ }^{27}$ Second, by assuming a shear-free $\mathrm{BC}$ over the gas/liquid regions, the viscous dissipation taking place in the underlying gas phase has been neglected. Both effects are expected to increase the surface friction, i.e., decrease the effective slip length. The results obtained in this section, therefore, provide an upper limit for the slip length. How curvature of the liquid-gas interface and dissipation in the gas phase affect the slip length will be discussed in Sec. III.

In the following, our aim is to define an effective boundary condition for the composite interface in the form of an effective (averaged) Navier BC:

$$
\left\langle\sigma_{w}\right\rangle=\eta_{l}\left\langle\dot{\gamma}_{w}\right\rangle=\lambda_{\text {eff }}\left\langle u_{w}\right\rangle,
$$

where $\eta_{l}$ is the liquid dynamic viscosity, $\lambda_{\text {eff }}$ is the effective surface friction coefficient, and $\left\langle\sigma_{w}\right\rangle,\left\langle\dot{\gamma}_{w}\right\rangle$, and $\left\langle u_{w}\right\rangle$ are, respectively, the averaged shear stress, shear rate, and (slip) velocity at the interface. This $\mathrm{BC}$ here expressed in the form of a stress balance at the interface can also be rewritten to introduce the effective slip length $b_{\text {eff }}$ characterizing the interface,

$$
b_{\text {eff }}\left\langle\dot{\gamma}_{w}\right\rangle=\left\langle u_{w}\right\rangle \quad \text { with } b_{\text {eff }}=\frac{\eta_{l}}{\lambda_{\text {eff }}} \text {. }
$$

Let us emphasize that the effective slip length $b_{\text {eff }}$ is the pertinent $\mathrm{BC}$ for the hydrodynamic problem at scales larger than those characterizing the underlying roughness $(L)$. The roughness scales are therefore integrated out in the definition of $b_{\text {eff. }}$.

\section{A. Limit of vanishing solid areas: $\phi_{s} \rightarrow 0$}

We first consider the case in which the solid fraction $\phi_{s}$ is very small. This is the interesting limit to obtain superlubricating surfaces, for which slippage effects are expected to be the largest.

In such a limit, the wall is almost frictionless, and close to the interface the flow is plug-like and described by the imposed plug-flow velocity $U$. Accordingly, the averaged slip velocity simply reads in this situation $\left\langle u_{w}\right\rangle \sim U$.

Let us now estimate the averaged viscous stress at the wall $\left\langle\sigma_{w}\right\rangle$ in this limit. The residual friction stress is only imposed on the solid parts, i.e., over a fraction $\phi_{s}$ of the surface. This leads to an averaged shear stress

$$
\left\langle\sigma_{w}\right\rangle=\phi_{s} \times \eta_{l}\left\langle\dot{\gamma}_{w}\right\rangle_{s},
$$

where $\left\langle\dot{\gamma}_{w}\right\rangle_{\mathrm{s}}$ is the local shear rate on the solid surface. To estimate this quantity, we recall that the Stokes equation has a Laplacian form, which strongly couples the spatial dependence of the velocity profile along the different axes $(x, y, z)$. This implies that $\left\langle\dot{\gamma}_{w}\right\rangle_{\mathrm{s}}=\left\langle\partial v_{x} / \partial z\right\rangle_{\mathrm{s}} \sim U / a$, with $a$ the typical size of the solid area.

Gathering the above results, one obtains that the effective slip length scales as $b_{\text {eff }} \sim a / \phi_{s}$. We thus write in the limit of small solid fraction $\phi_{s}$ 


$$
b_{\text {eff }} \underset{\phi_{s} \rightarrow 0}{\sim} \alpha \frac{a}{\phi_{s}}
$$

with $\alpha$ a numerical prefactor, which is expected to depend on the underlying geometry of the surface (stripes, posts, etc.). This relationship is the main result of this paper.

First, it is interesting to compare this scaling law (4) to existing analytical predictions in simple geometries. For periodic grooves oriented parallel ${ }^{8,9}$ (respectively, perpendicular $^{24}$ ) to the flow, the exact expression for $b_{\text {eff }}$ actually reads

$$
b_{\text {eff }}=\frac{-L}{\pi} \log \left[\cos \left(\frac{\pi}{2}\left(1-\phi_{s}\right)\right)\right]
$$

[respectively, $1 / 2$ of Eq. (5)]. In the limit of vanishing solid fraction $\phi_{s}$, it therefore predicts $b_{\text {eff }}$ to depend only logarithmically on $\phi_{s}$ through $b_{\text {eff }} \sim-L \log \phi_{s}$. For this groove geometry, the solid fraction simply reads $\phi_{s}=a / L$ so that the scaling law approach (4) now becomes

$$
b_{\text {eff }} \underset{\phi_{s} \rightarrow 0}{\sim} L .
$$

In agreement with the exact calculation, our approach, therefore, predicts no dependency of the effective slip length on the solid fraction $\phi_{s}$ to leading order in $\phi_{s}$ (i.e., up to the logarithmic term).

We now consider a more complex geometry of major practical interest: a bidimensional (2D) pattern of posts. In this situation, the solid fraction now reads $\phi_{s}=(a / L)^{2}$ so that Eq. (4) predicts that

$$
b_{\text {eff }} \underset{\phi_{s} \rightarrow 0}{\sim} \alpha \frac{L}{\sqrt{\phi_{s}}} .
$$

Since no exact calculation is available for post patterns, we have checked the validity of the above scaling law using a numerical calculation for the slip length. To this end, we used a previously reported numerical approach. ${ }^{25}$ We only briefly recall here the basic steps of this approach and refer the reader to Ref. 25 for a more detailed description. A shear flow is considered over a composite surface characterized by a heterogeneous slip length pattern. The boundary is modeled by a pattern of local slip lengths on a planar surface. The characteristics of the flow far away from the surface and an effective slip length are determined by solving the hydrodynamic equations with the hydrodynamic BC given by the local slip length, using an integral method.

Using this numerical approach, the effective slip length was computed for a square lattice of solid patches of square cross section. The computed slip lengths $b_{\text {eff }} / L$ are plotted in Fig. 2 against $1 / \sqrt{\phi_{s}}$ for different solid fractions in the range $\phi_{s}<30 \%$. The agreement with the prediction (7) is shown to be excellent, therefore validating the proposed scaling law. As an additional check, these results were complemented by numerical results obtained from 3D finite-element methods (see Sec. III B for details) in the same geometry (square lattice of solid patches of either square or disk shape). As shown in Fig. 2, the agreement is again excellent, with all data collapsing on a single straight line. A linear regression performed on the numerical calculation data allows us to

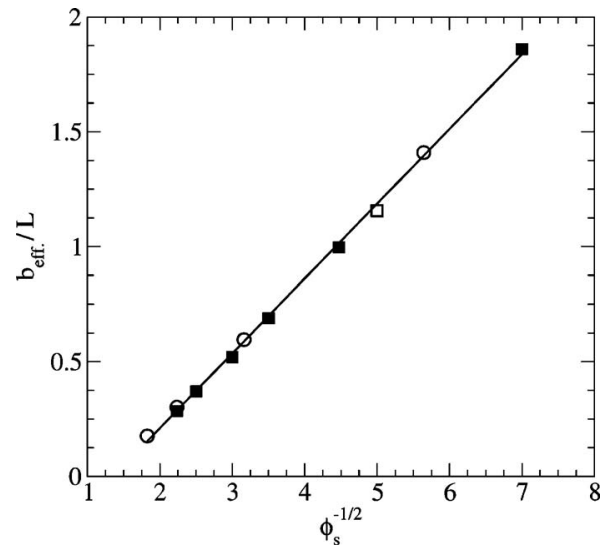

FIG. 2. Normalized effective slip length $b_{\text {eff }} / L$ for a composite surface made of solid patches organized on a square lattice; limit of low solid fraction: $\phi_{s}=2-30 \%$. $b_{\text {eff }} / L$ is plotted against $1 / \sqrt{\phi_{s}}$ according to Eq. (4) with $\phi_{s}$ $=(a / L)^{2}$. 2D numerical approach (Ref. 25): (ם) solid patches of square cross section; 3D finite-element calculation: solid patches of ( $\square$ ) square cross section, $(\bigcirc)$ circular cross section; (-) linear regression: $b_{\mathrm{eff}} / L$ $=0.325 / \sqrt{\phi_{s}}-0.44$.

access the coefficients of the scaling behavior, Eq. (7), $b_{\text {eff }} / L \simeq 0.325 / \sqrt{\phi_{s}}-0.44$. This formula provides a useful and very simple expression for the slip length on patterned surfaces of posts.

To finish, we show that it is simple to relax the assumption of a no-slip BC on the solid areas, assuming a finite intrinsic slip length $b_{s}$. Slippage over a bare, smooth surface (here denoted as "intrinsic slippage") has been intensively investigated over the past decade. ${ }^{28}$ An intrinsic slip length $b_{s}$ of a few tens of nanometers is demonstrated over smooth, hydrophobic surfaces. ${ }^{20-23}$

Going back to the above derivation of the scaling law in the limit $\phi_{s} \rightarrow 0$, one expects that a finite slip length on the solid will reduce the shear rate $\left\langle\dot{\gamma}_{w}\right\rangle_{s}$ over the solid regions: $\left\langle\dot{\gamma}_{w}\right\rangle_{s} \sim U /\left(a+b_{s}\right)$. The averaged shear stress over the total surface now reads $\left\langle\sigma_{w}\right\rangle=\phi_{s} \eta_{l} U /\left(a+b_{s}\right)$. One gets accordingly a modified scaling law for the effective slip length,

$$
b_{\mathrm{eff}} \underset{\phi_{s} \rightarrow 0}{\sim} \frac{a+b_{s}}{\phi_{s}} .
$$

This modified scaling is tested on the square lattice geometry of solid patches in Fig. 3. The difference between the effective slip length $b_{\text {eff }}\left(\phi_{s}, a, b_{s}\right)$ with intrinsic slippage $b_{s}$ on the solid, and the no-slip prediction (ideal case shown in Fig. 2) $b_{\text {ideal }} \equiv b_{\text {eff }}\left(\phi_{s}, a, b_{s}=0\right)$, is plotted against $b_{s} / \phi_{s}$. This figure confirms the soundness of the scaling law (8), which, therefore, allows us to quantify the impact of an intrinsic solid slip on the overall effective slip over superhydrophobic surfaces.

Additionally, it is important to note that $b_{s} / \phi_{s}$ does not depend on the geometry of the surface so that the increase of the effective slip length due to the intrinsic slip is independent of the details of the underlying surface texture (stripes or posts). Moreover, Eq. (8) shows that such a term will play a role only if the size of the solid region, $a$, is of the order of the intrinsic slip length: $b_{s} \sim a$. With $b_{s}$ limited to a few tens 


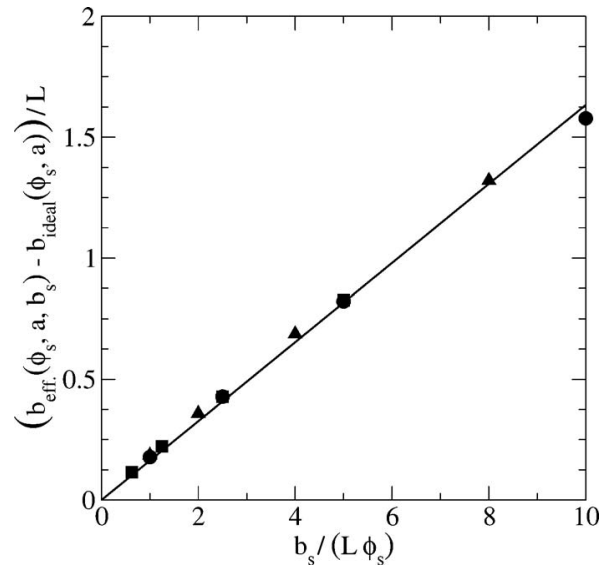

FIG. 3. Influence of a finite slippage on solid patches on the normalized effective slip length $b_{\text {eff }} / L$ for a composite surface made of solid patches organized on a square lattice; limit of low solid fraction: $\phi_{s}=2-16 \%$. Increase in $b_{\text {eff }} / L$ compared to the reference for no slip on solid is plotted against $b_{s} /\left(L \phi_{s}\right)$ according to Eq. (8). 2D numerical approach for patches of square cross section (Ref. 25): (৩) $\phi_{s}=2 \%,(\mathbf{\Delta}) \phi_{s}=5 \%,(\boldsymbol{\square}) \phi_{s}=16 \%$; (一) linear regression: $\Delta b_{\text {eff }} / L=0.165 b_{s} /\left(L \phi_{s}\right)$.

of nanometers, ${ }^{28}$ intrinsic slippage will only play a role for very sharp nanostructures, with $a$ in the tens of nanometer range.

\section{B. Limit of vanishing gas areas: $\phi_{s} \rightarrow 1$}

We now consider the opposite limit of vanishing gas area: $\phi_{s} \rightarrow 1$. This limit has a much lower practical importance than the previous one, $\phi_{s} \rightarrow 0$. Indeed, in this case the shear-free area will have a tiny influence on the wall friction and the effective slippage is expected to be much smaller. We mention this result for completeness.

Since in this limit the BC is almost everywhere a no-slip $\mathrm{BC}$, the flow is now appropriately described by the imposed shear rate $\dot{\gamma}$. Accordingly, the averaged viscous stress at the wall is expected to basically reduce to $\left\langle\sigma_{w}\right\rangle \simeq \eta_{l} \dot{\gamma}$ in this limit.

Now, the averaged slip velocity at the wall only results from the $\left(1-\phi_{s}\right)$ surface fraction of gas areas. In these regions, the typical fluid velocity is of order $\ell \dot{\gamma}$, with $\ell$ a characteristic length scale for the gas areas. Combining those two estimates leads to the scaling behavior

$$
b_{\text {eff }} \underset{\phi_{s} \rightarrow 1}{\sim} \ell\left(1-\phi_{s}\right) .
$$

Again, this result (9) can be first compared with analytical predictions for grooves. ${ }^{8,9,24}$ For this simple one-dimensional geometry (1D), the typical length scale $\ell$ is simply $\ell=L(1$ $\left.-\phi_{s}\right)$ and Eq. (9) reduces to $b_{\text {eff }} \sim L\left(1-\phi_{s}\right)^{2}$. This prediction is in perfect agreement with the analytic expression in this limit. ${ }^{8,9,24}$

Turning now to geometries made of $2 \mathrm{D}$ patterns, the scaling law predictions are tested against the numerical results for the slip length obtained using the approach described in Ref. 25. Two geometries have been considered: a square lattice of square solid patches, corresponding to a

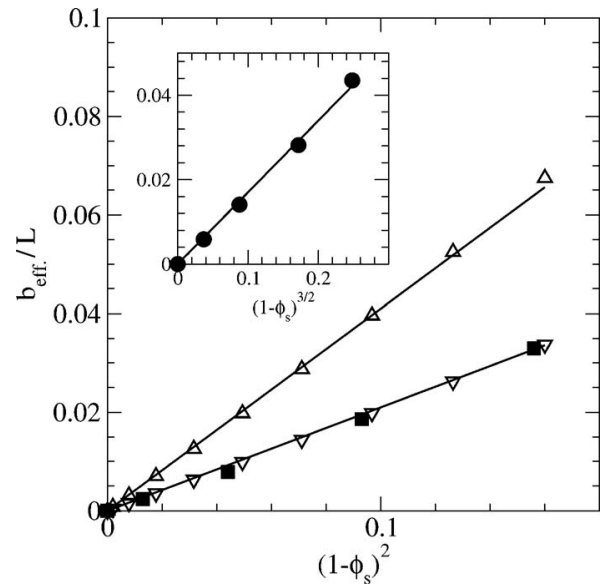

FIG. 4. Normalized effective slip length $b_{\text {eff }} / L$ for a composite surface of various geometries in the limit of high solid fraction: $\phi_{s}=60-100 \% . b_{\text {eff }} / L$ is plotted against $\ell\left(1-\phi_{s}\right) / L$ according to Eq. (9) with $\ell$ the geometrydependent typical size of shear-free areas. Symbols are numerical data obtained by solving the hydrodynamics equations (Ref. 25). Main: $\ell=L(1$ $-\phi_{s}$ ); (Ш) square solid patches organized on a square lattice (surface structure made of pillars), $(\nabla)$ solid stripes perpendicular to the flow, $(\triangle)$ solid stripes parallel to the flow, (-) linear regressions: slopes are, respectively, $0.21 \pm 0.02$ and $0.41 \pm 0.04$. Inset: $\ell=L \sqrt{1-\phi_{s}} ;(\bullet)$ square shear-free patches organized on a square lattice (surface structure made of holes), (-) linear regression: slope is 0.17 .

surface made of pillars as already studied in Sec. II A, and of square gas (shear-free) patches, corresponding to a holes surface texture.

It is interesting to note that the two $2 \mathrm{D}$ geometries investigated do not behave the same way in the considered limit (a point discussed in more detail in Sec. II C). Namely, for the hole pattern, the characteristic size $\ell$ simply relates to the area of the hole so that one has $\ell=L \sqrt{1-\phi_{s}}$. On the contrary, for a post pattern, the characteristic size $\ell$ over which the flow is modified is associated with the small width of the gaseous stripe separating two adjacent posts. Consequently, $\ell$ scales as $\ell \sim L\left(1-\phi_{s}\right)$ for the post pattern, which means that this $2 \mathrm{D}$ structure behaves as a stripe-like geometry when $\phi_{s} \rightarrow 1$.

Numerical results for the effective slip length obtained with the different geometries (simple grooves, posts, and holes) are gathered in Fig. 4. The main figure shows data obtained for grooves-either parallel or perpendicular to the flow-and posts. The expected scaling $b_{\text {eff }} \simeq \alpha L\left(1-\phi_{s}\right)^{2}$ is very well verified with $\alpha=0.41 \pm 0.04$ (respectively, $\alpha$ $=0.21 \pm 0.02$ ) agreeing with the prefactor $\alpha=0.393$ (respectively, 0.186) obtained from analytical prediction, ${ }^{8,9,24}$ for grooves parallel (respectively, perpendicular) to the flow [see Eq. (5)]. As predicted, in this $\phi_{s} \rightarrow 1$ limit, the post geometry is equivalent to perpendicular grooves in term of surface frictional properties.

Finally, the inset of Fig. 4 displays data for the hole pattern. The modified behavior now predicted by the scaling law, $b_{\text {eff }} \simeq \alpha L\left(1-\phi_{s}\right)^{3 / 2}$, is perfectly recovered.

Overall, the agreement with Eq. (9) is shown to be excellent for all geometries and for solid fractions $\phi_{s}>60 \%$.

Eventually, we quote that the effect of intrinsic slippage on the solid surface can be considered along the same lines 
as above, yielding the prediction $b_{\text {eff }} \sim b_{s}$ to leading order in $\left(1-\phi_{s}\right)$ : the main contribution to slippage originates in the intrinsic slippage on the solid surface, therefore corresponding to a small effective slip length.

\section{Influence of the geometry on the effective slip length}

In the experimentally interesting limit $\phi_{s} \rightarrow 0$, the different results can be summarized, restricting to the case of a no-slip $\mathrm{BC}$ on the solid regions, as

$$
\begin{aligned}
& \text { stripe geometry } b_{\text {eff }} \sim-L \log \left(\phi_{s}\right), \\
& \text { post geometry } b_{\text {eff }} \sim \frac{L}{\sqrt{\phi_{s}}} .
\end{aligned}
$$

These predictions show that the geometry has a strong effect on the effective slip length. Indeed, for stripe geometries the log-dependency in $\phi_{s}$ practically limits the achievable effective slip lengths to no better than the surface periodicity $L$. On the contrary, the power-law divergence exhibited by the post pattern as $\phi_{s} \rightarrow 0$ makes it possible to overcome the $L$ bound to achieve much higher effective slip lengths.

It is interesting to note that this change in behavior does not hold for any $2 \mathrm{D}$ pattern as opposed to a $1 \mathrm{D}$ pattern. As already noticed in the preceding section, 2D holes and post patterns may have completely different frictional properties, and in the present limit $\phi_{s} \rightarrow 0$, the pattern of holes surface indeed reduces to a stripe-like one. As a matter of fact, for holes, $\phi_{s} \simeq 2 a / L$ (with $a$ the thickness of the solid walls surrounding the holes), so that the general predictions, Eq. (4), lead to $b_{\text {eff }} \sim L$, and holes thus behave like stripes.

Of course, this is to compare with the opposite limit $\phi_{s} \rightarrow 1$, where we already noticed that the post pattern this time behaved as a stripe-like structure, in contrast to the behavior of the hole pattern.

The two limiting cases can be rationalized in the following way. Slippage in the $\phi_{s} \rightarrow 0$ limit depends on the typical scale $a$ of the solid phase [see Eq. (4)], while in the $\phi_{s} \rightarrow 1$ limit it depends on the gas phase extension $\ell$ [see Eq. (9)]. A structure shifts from what we called 2D behavior to a 1D stripe-like behavior whenever this typical size $a$ (respectively, $\ell$ ) no longer reduces to the square root of the solid (respectively, gas) phase area. Not surprisingly, this type of criterion amounts to considering a shape factor for the phase under scrutiny: an elongated phase structure leading to a stripe-like behavior.

These points are summarized in Fig. 5, where the effective slip lengths are plotted as a function of the gas area fraction $\left(1-\phi_{s}\right)$ for different patterns: parallel grooves, perpendicular grooves, pillars, and holes. Note that for discretization reasons in the numerical calculations, we were limited to solid fractions $\phi_{s}$ higher than $1 \%$ for pillars, and to the more stringent condition $\phi_{s} \geqslant 10 \%$ for holes. Even with this restriction, the effective slip length for surfaces made of pillars (squares) is seen to be much higher than for periodic grooves (solid and dashed lines) and holes (circles) in the limit $\phi_{s} \rightarrow 0$. Additionally, one verifies that holes behave as grooves perpendicular to the flow in this low solid fraction

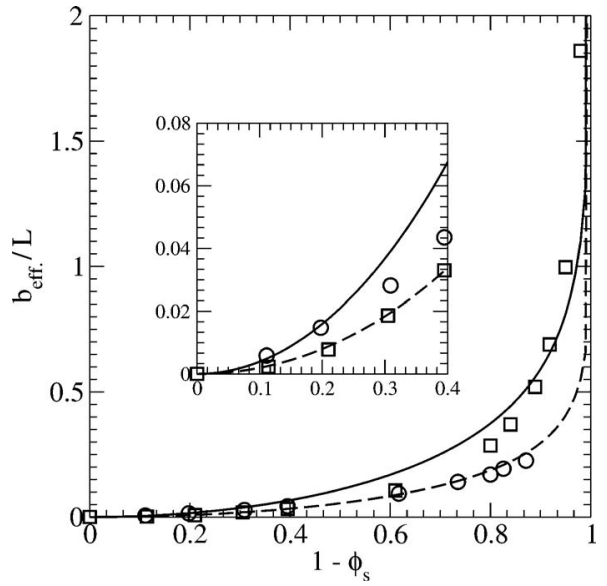

FIG. 5. Normalized effective slip length $b_{\text {eff }} / L$ as a function of the gas area fraction $\left(1-\phi_{s}\right)$ for a composite surface of geometries. 2D numerical approach (Ref. 25) ( $\square$ ) square solid patches organized on a square lattice (surface structure made of pillars), $(\mathrm{O})$ square shear-free patches organized on a square lattice (surface structure made of holes), (--) solid stripes perpendicular to the flow, (-) solid stripes parallel to the flow. Inset: focus on the low gas fraction region.

limit, while in the opposite limit (shown in the inset of Fig. 5 ), it is the pillar surface that behaves that way. In the limit $\phi_{s} \rightarrow 1$, the surface made of holes is the one having the higher effective slip length for a given solid fraction.

Altogether, surfaces made of pillars (posts) are the best candidates to obtain very large slip lengths in the $\phi_{s} \rightarrow 0$ limit.

\section{TOWARD A GENERAL DESCRIPTION FOR THE SLIP LENGTH}

In the preceding section, we derived general scaling laws relating the surface geometry to the effective surface friction at the superhydrophobic wall. However, these general scaling laws are obtained within an idealized description assuming a flat liquid interface and "ideal" shear-free $\mathrm{BC}$ over the gaseous regions.

The validity of these assumptions is now examined and these effects are quantified. Expressions for the effective slip lengths taking these effects into account are provided as a predictive tool for experimental situations.

\section{A. An interpolation formula for composite slippage}

We start by considering the situation in which the slip length on the gas phase is not infinite, so that the local $\mathrm{BC}$ is not a shear-free $\mathrm{BC}$ as assumed in Sec. II. We denote as $b_{g}$ the slip length on the liquid-gas interface. We assume that a no-slip BC applies on the solid regions.

Let us first consider the limiting case $b_{g} \rightarrow 0$. For a shear flow with shear rate $\dot{\gamma}$, the velocity profile may be approximated as $u(x, y, z)=\dot{\gamma}[z+b(x, y)]$, with $b(x, y)$ the locally varying slip length. Such an approximation is expected to be valid when $b$ is smaller than its variation length scale $(\nabla b$ $\ll 1$, expressed here as $\left.b_{g} \ll L\right)$. The averaged velocity profile thus reads $\langle u\rangle(z)=\dot{\gamma}(z+\langle b\rangle)$. Using the definition of the ef- 


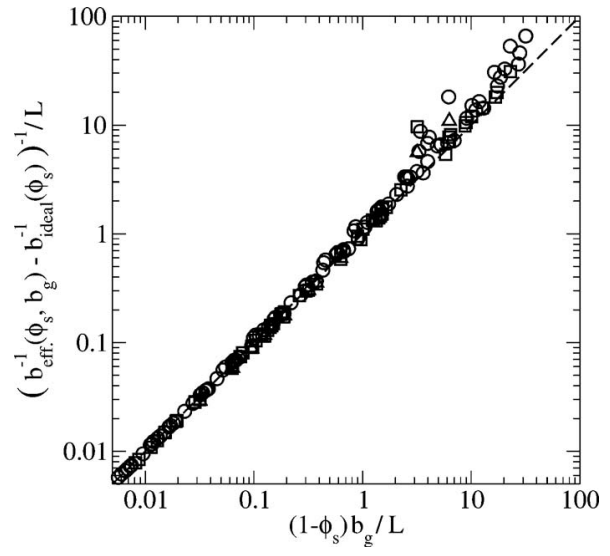

FIG. 6. Effective slip length $b_{\text {eff }}$ for various surface geometries, compared with the interpolation formula (12). Numerical results for the effective slip length using the approach in Ref. 25: $(\square)$ periodic pattern of pillars with $\phi_{s}=3-80 \% ;(\triangle)$ periodic pattern of holes with $\phi_{s}=60-80 \%$. This also incorporates the effect of the viscosity of the gas phase $\eta_{g}$ on the effective slip length (see Sec. III B). In this case, the effective slip length is calculated using finite-element calculations with various surface heights $e / L[0.05-2]$ and viscosity ratio $\eta_{l} / \eta_{g}:[0.01-100]:(\bigcirc, \bullet)$ stripes (perpendicular or parallel to the flow) with $\phi_{s}=20 \%$; $(\square)$ same for pillars; $(\triangle)$ same for holes. (--) linear prediction: $y=x$.

fective slip length, this velocity profile is expected to identify with $\langle u\rangle(z)=\dot{\gamma}\left(z+b_{\text {eff }}\right)$. This yields the following expression for the effective slip length in this limit:

$$
b_{\text {eff }}=\left(1-\phi_{s}\right) b_{g} \text {. }
$$

One may show this result more rigorously using a perturbative approach of the flow field in $b_{g}$. The reference flow field corresponds to $b_{g}=0$, which reads, for a shear rate $\dot{\gamma}$ at infinity, $u_{0}=\dot{\gamma} z$. The first-order correction for the flow field over a composite surface with no-slip region $S_{0}$ and partial slip region $S_{g}$ thus verifies $\eta_{l} \Delta \mathbf{u}_{1}=\nabla P_{1}$, together with the following boundary condition on the liquid-gas interface: $\left.\quad b_{g} \partial_{z}\left(\mathbf{u}_{0}+\mathbf{u}_{1}\right)\right|_{w}=\left.\mathbf{u}_{1}\right|_{w}$. In the limit $b_{g} \rightarrow 0$, one has $\left.u_{1}\right|_{w} \simeq b_{g} \dot{\gamma}$ so that in the end $\left\langle u_{w}\right\rangle=\left(1-\phi_{s}\right) b_{g} \dot{\gamma}$, and $b_{\text {eff }}=\left(1-\phi_{s}\right) b_{g}$ as shown above.

For large $b_{g}$, no such simple prediction could be obtained. However, it is possible to propose an interpolation formula that will prove to be useful in the following. This interpolation is based on the two known limits: for $b_{g} \rightarrow 0$, $b_{\text {eff }}=\left(1-\phi_{s}\right) b_{g}$; for $b_{g} \rightarrow \infty, b_{\text {eff }} \equiv b_{\text {ideal }}$ with $b_{\text {ideal }}$ the effective slip length obtained in the idealized case where a shearfree $\mathrm{BC}$ is assumed at the liquid/gas interface. $b_{\text {ideal }}$ is given by the expressions discussed in Sec. II (typically $b_{\text {ideal }}$ $\sim a / \phi_{s}$ ).

A heuristic formula interpolating between these two limiting cases is

$$
\frac{1}{b_{\text {eff }}}=\frac{1}{\left(1-\phi_{s}\right) b_{g}}+\frac{1}{b_{\text {ideal }}} .
$$

This amounts to adding the friction coefficient in the above two limits. This interpolation formula is tested in Fig. 6. While there is no deep physical justification for such a formula, it is shown to provide a very good description of the effect of a finite slip length on the liquid-gas interface.
In the following, we will make use of this interpolation to discuss the effect of a finite dissipation in the gas phase.

\section{B. Finite dissipation within the gas subphase}

We now discuss how the dissipation in the gas phase modifies the friction properties and the effective slip length.

In Sec. II, the assumption of a shear-free BC at the liquid-gas interface implicitly amounts to neglecting such a dissipation. As a consequence, the predicted effective slip length at the surface does not depend on the liquid viscosity. This is to contrast with the opposite situation, which we will refer to as the "gas cushion model," which assumes that the dissipation at the surface is dominated by the shearing of a continuous gas layer, lying in between the solid and liquid phases (gas cushion). Such a model predicts ${ }^{29,30}$

$$
b_{\text {eff }}=\frac{\eta_{l}}{\eta_{g}} e,
$$

with $\eta_{g}$ the gas dynamic viscosity and $e$ the thickness of the gas layer. Of course in the limit of vanishing $\phi_{s}$, this should represent an upper bound value toward which the slip length will saturate.

In the following, we develop an approach to estimate the dissipation resulting from the gas phase in intermediate cases (finite $\phi_{s}$ and $\eta_{g} / \eta_{l}$ ). Focusing on a surface structure corresponding to a square lattice of cylindrical pillars, which resemble most of the artificial superhydrophobic surfaces, we will first define and estimate an averaged slip length $b_{g}$ over the gas area. Then we will make use of the interpolation formula, Eq. (12), to obtain the effective slip length.

Before proceeding with this strategy, it should be mentioned that the problem of Stokes shear flow over a liquidimpregnated roughness $\left(\eta_{g}\right.$ now equals $\left.\eta_{l}\right)$ has been the subject of different works, especially in the simpler groove geometry, where it has been solved analytically by Wang ${ }^{31}$ using eigenfunction expansions and matching. In this simpler geometry, a strategy might then be to follow Wang's approach by modifying the matching condition to incorporate the viscosity contrast. To our knowledge, however, no such route has been developed for the surfaces made of pillars that we consider now.

We start with the estimate of the averaged slip length $b_{g}$ over the gas area. To evaluate the extra dissipation occurring within the gas subphase, we use an effective medium approach analogous in spirit to Darcy-Brinkmann's law in a 2D porous medium. ${ }^{32}$ The gas is assumed to flow parallel to the bottom reference surface (see Fig. 1) and satisfies a Stokes equation,

$$
\eta_{g} \Delta U_{\|}=\nabla_{\|} P,
$$

with $P$ the gas pressure. An effective medium approach amounts to replacing the (gas + pillars) problem by a homogeneous problem with an effective velocity $\widetilde{U}_{\|}$and an effective pressure gradient whose strength is set by the drag on the pillars, 


$$
-\nabla_{\|} \widetilde{P}=\Sigma F_{d},
$$

with $\Sigma=1 / L^{2}$ the number density of pillars and $F_{d}$ the drag force per unit of length experienced by a single pillar. The latter is of the form $F_{d}=-\xi\left(\phi_{s}\right) \tilde{U}_{\|}$, where $\xi\left(\phi_{s}\right)$ is the friction coefficient, the $\phi_{s}$ dependency of which accounts for hydrodynamic interactions between pillars and can be found in the literature. ${ }^{33}$ For example, for a square array of pillars, a series expansion in $\phi_{s}$ leads to

$$
\begin{aligned}
\frac{4 \pi \eta_{g}}{\xi\left(\phi_{s}\right)}= & -\frac{1}{2} \ln \phi_{s}-0.738+\phi_{s}-0.887 \phi_{s}^{2}+2.039 \phi_{s}^{3} \\
& +O\left(\phi_{s}^{4}\right),
\end{aligned}
$$

accurate to within 5\% in the range of practical interest $\phi_{s}$ $<30 \%$. Combining Eqs. (14) and (15) with the definition of $\xi\left(\phi_{s}\right)$, one obtains the relation

$$
\Delta \widetilde{U}_{\|}=\frac{\sum \xi\left(\phi_{s}\right)}{\eta_{g}} \widetilde{U}_{\|}
$$

complemented by the BCs $\widetilde{U}_{\|}(z=0)=\left\langle u_{w}\right\rangle_{g}$ and $\tilde{U}_{\|}(z=-e)$ $=0$. It resolves straightforwardly and yields an averaged viscous surface stress of

$$
\left\langle\sigma_{w}\right\rangle_{g}=\eta_{g}\left\langle u_{w}\right\rangle_{g} \frac{q}{\tanh q e},
$$

with $q^{2}=\Sigma \xi\left(\phi_{s}\right) / \eta_{g}$. According to Eqs. (1) and (2), this predicts for the averaged slip length $b_{g}$ over the gas areas

$$
b_{g}=\frac{e \eta_{l}}{\eta_{g}} \frac{\tanh q e}{q e},
$$

with $q\left(\phi_{s}\right)$ given above, an expression reminiscent of the Beavers and Joseph BC on porous media. ${ }^{34}$

To estimate the effective slip length, we now use this prediction together with the interpolation formula, Eq. (12), to obtain

$$
\frac{1}{b_{\text {eff }}}=\frac{1}{\left(1-\phi_{s}\right) \frac{e \eta_{l}}{\eta_{g}} \frac{\tanh q e}{q e}}+\frac{1}{b_{\text {ideal }}},
$$

where we recall that $b_{\text {ideal }}$ stands for the effective slip length for the composite surface with a negligible dissipation in the gas (shear-free BC), as discussed in Sec. II.

To assess the validity of this approach, we have conducted numerical resolution of the Stokes equations using finite-element methods implemented through $\mathrm{Comsol}^{\circledR}$. The computed geometry is like the one sketched in Fig. 1 with an undeformable planar liquid interface separating a liquid phase and a gas phase, with a given viscosity ratio $\eta_{g} / \eta_{l}$. A planar upper wall (not sketched) encloses the liquid slab separated from the liquid interface by a distance $H$ ( $H$ was chosen large enough so that the results matched the $H \rightarrow \infty$ limit). Symmetries were used to reduce the size of the computation cell. A Couette flow is simulated by imposing a velocity $U$ of the top planar wall.

The resulting flow field is computed, from which we have extracted the effective slip length $b_{\text {eff }}$ over the entire surface, as done previously. We also measured the averaged

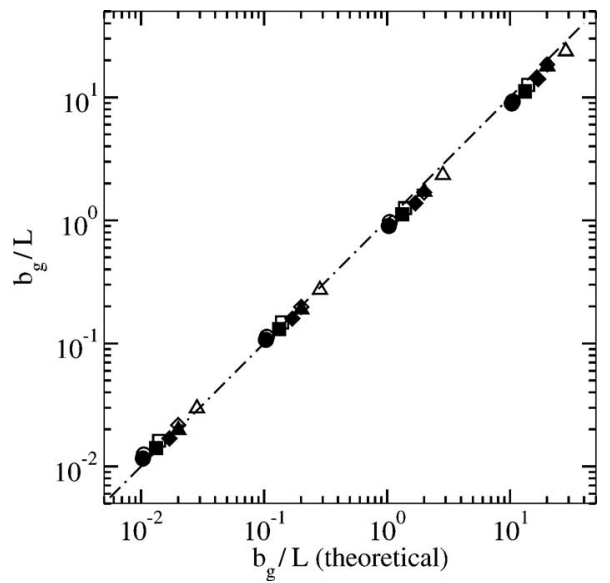

FIG. 7. Effective slip length $b_{g}$ over gas areas as obtained from finiteelement calculations, compared with theoretical predictions according to Eq. (19). The surface is made of cylindrical pillars of height $e$ arranged on a square lattice and the viscosity ratio $\eta_{l} / \eta_{g}$ between the two phases is varied from 0.1 to 100 ; solid and empty symbols correspond, respectively, to $e / L$ $=0.25$ and $e / L=1$. $(\bigcirc, \bigcirc) \phi_{s}=30 \%,(\square, \square) \phi_{s}=20 \%,(\diamond, \diamond) \phi_{s}=10 \%$, $(\triangle, \mathbf{\Delta}) \phi_{s}=3 \%,(-\cdot)$ perfect match: $y=x$.

slip length $b_{g}$ over the gas areas. The latter is defined from the averaged shear stress $\left\langle\sigma_{w}\right\rangle_{g}$ and the averaged slip velocity $\left\langle u_{w}\right\rangle_{g}$ over the gas phase according to Navier-like relationships of the form (1) and (2).

Let us first discuss the result for the averaged slip length $b_{g}$. The comparison between the theoretical prediction (19) and the numerical results using finite-element computations is shown in Fig. 7. For the explored viscosity ratios $\eta_{l} / \eta_{g}$, the gas layer thickness $e / L$, and the solid fraction $\phi_{s}$, our analytical prediction proves very satisfactory and remains always within $20 \%$ of the numerical value.

We can now test the global result for the effective slip length, in Eq. (20). This phenomenological relationship is tested by comparing with the results of the 3D finite-element calculation-where both $b_{\text {eff }}$ and $b_{g}$ are measured-but also by comparing with the $2 \mathrm{D}$ numerical approach ${ }^{25}$ with a flat composite interface with mixed BC: no-slip and partial slip with slip length $b_{g}$. This is done in Fig. 6 , where $\left(b_{\text {eff }}^{-1}\right.$ $\left.-b_{\text {ideal }}^{-1}\right)^{-1}$ is plotted against $\left(1-\phi_{s}\right) b_{g}$. As already noted, the interpolating formula (20) appears to be astonishingly efficient. It describes with very good precision the effect of the finite dissipation within the underneath subphase. Combining Eqs. (16) and (20) allows us to quantify the influence of the subphase dissipation on the effective slip length.

\section{Pressure dependency: Meniscus curvature effect}

In this section, we discuss qualitatively the effect of a pressure drop $\Delta P=P_{g}-P_{l}$ between the liquid and the underlying gas phase. A pressure drop is associated with a curvature of the liquid-vapor meniscus, leading to a reduction of slippage. Our aim in this section is to provide an upper limit for the pressure drop $\Delta P$ below which curvature effects can be neglected. To this end, we shall focus on the case in which the gas meniscus is oriented outward (protruding by a distance $\delta$ into the liquid phase), which can be handled in a simple way within the present description. This situation was 
recently shown to be relevant experimentally whenever no direct control can be exerted on the gas subphase, ${ }^{35}$ and we expect the present analysis to capture the general features occurring at curved interfaces. ${ }^{27,36}$

To obtain how pressure-induced curvature effects modify the slip length, we follow a similar approach to that in the preceding section. The additional dissipation resulting from the boundary curvature will be incorporated as a finite slip length $b_{g}$ applied on the liquid-gas interface. In the case of a curved gas meniscus, this effective slip length $b_{g}$ should correspond to the local radius of curvature, ${ }^{37}$ which reads $b_{g} \sim \ell^{2} / \delta$ in a parabolic approximation.

This relationship, based on the local expression of the stress tensor in the tangential/normal vector frame, ${ }^{37}$ can actually be recovered with a simple scaling argument. Starting from the expression for the drag force $F=4 \pi \eta_{l} R u$ experienced by a spherical bubble moving at velocity $u,^{38,39}$ we expect the additional drag exerted on the cap to be of the form

$$
\langle F\rangle_{g} \sim \eta_{l} \delta\left\langle u_{w}\right\rangle_{g} .
$$

The averaged shear stress resulting from this drag force is simply $\left\langle\sigma_{w}\right\rangle_{g}=\langle F\rangle_{g} / \ell^{2}$ with $\ell$ the typical length scale for the gas areas so that in the end one recovers for the effective slip length over the gas areas

$$
b_{g} \sim \ell^{2} / \delta \text {. }
$$

We are now in a situation similar to the one considered in Sec. III B except for the origin of $b_{g}$. The effective slip length can be obtained using the interpolation formula (12).

It is first interesting to compare this prediction with a recent analytical work considering the 1D situation of a shear flow parallel to grooves. ${ }^{36}$ In this geometry, a first-order perturbative approach was developed that allowed to quantify the effect of meniscus curvature in the limit $\delta \ll \ell(\ell=L$ $-a)$. Considering the limit of large solid fraction $\phi_{s}$, our scaling approach provides in this 1D situation the following curvature correction to the effective slip length:

$$
b_{\text {eff }}-b_{\text {ideal }} \sim-\left(1-\phi_{s}\right) \delta,
$$

which is obtained by combining Eqs. (9), (12), and (22). This result agrees fully with the first-order correction term found in Ref. 36.

In the more interesting limit of a 2D surface pattern with $\phi_{s} \rightarrow 0$, the ideal flat surface slip length is now given by Eq. (4). We thus obtain

$$
b_{\text {eff }}=\left(\frac{\delta}{L^{2}}+\frac{1}{b_{\text {ideal }}}\right)^{-1}
$$

with $b_{\text {ideal }} / L \simeq 0.325 / \sqrt{\phi_{s}}-0.44$ (see Sec. II). This result shows that in the limit $b_{g}=L^{2} / \delta<b_{\text {ideal }}$, the effective slip length $b_{\text {eff }}$ does saturate at the gas spherical cap radius of curvature. Coming back to macroscopic variables, this radius of curvature can be traced back to the pressure difference between the liquid and the gas phase through the Laplace equation, $\Delta P=P_{g}-P_{l}=2 \gamma_{\mathrm{lg}} \delta / L^{2}$, with $\gamma_{\mathrm{lg}}$ the liquid/gas surface tension. A pressure difference between the liquid and the gas thus induces a saturation of the effective slip length at a
TABLE I. Summary of the effective slip length for flows over a surface made of pillars in various situations. $b_{g}$ origin and expressions: (a) sublayer viscous dissipation $b_{g}=\left(e \eta_{l} / \eta_{g}\right) \tanh (q e) /(q e)$; (b) curvature of menisci $b_{g}$ $=2 \gamma_{l v} / \Delta P$.

\begin{tabular}{ll}
\hline \multicolumn{1}{c}{ Model assumptions } & \multicolumn{1}{c}{$b_{\text {eff }}$} \\
\hline Ideal: flat, no-slip, shear-free & $b_{\text {ideal }}=L\left(0.325 / \sqrt{\phi_{s}}-0.44\right)$ \\
Finite solid slip $b_{s}$ & $b_{\text {ideal }}+0.165 b_{s} / \phi_{s}$ \\
Finite gas slip $b_{g}^{(\mathrm{a}),(\mathrm{b})}$ & $\left\{b_{\text {ideal }}^{-1}+\left[\left(1-\phi_{s}\right) b_{g}\right]^{-1}\right\}^{-1}$ \\
\hline \hline
\end{tabular}

value $b_{\text {eff }} \rightarrow 2 \gamma_{\mathrm{lg}} / \Delta P$. To fix ideas, for $\Delta P \sim 1$ bar, $b_{\text {eff }}$ saturates at $b_{\text {eff }} \sim 1 \mu \mathrm{m}$.

These results allow us to estimate the domain of pressure difference $\Delta P$, for which the curvature effects do not affect the slippage on the superhydrophobic surface. According to Eq. (24), this amounts to $L^{2} / \delta \gg b_{\text {ideal }}$, with $b_{\text {ideal }}$ the slip length when curvature effects are negligible. This can be rewritten

$$
\Delta P \ll \frac{\gamma_{\mathrm{lg}}}{b_{\text {ideal }}} .
$$

This condition shows that situations with larger slip lengths are more sensitive to curvature effects. However, on can note that a reasonable pressure difference in the order of tens of mbars will not affect a slip length in the hundreds of micrometers range. It should therefore be moderately limiting in the design of reduced friction interfaces. This is supported by the absence of pressure effects reported in Ref. 19 or the low curvature reported in Ref. 13.

We eventually remark that such curvature effects are expected to be more important for surfaces with a roughness made of holes, for which the gas is not connected to a reservoir. Depending on the condition for surface immersion, gas may be trapped in the holes with a non-negligible excess pressure. Such effects have indeed been observed in Ref. 35, with a geometry of holes of size $\ell \sim 650 \mathrm{~nm}$, thus resulting in a strongly reduced slip length.

\section{DISCUSSION}

The results obtained in the previous sections are summarized in Table I for the pillars' surface geometry. Based on this, we now discuss how to optimize slippage, and what maximum slip length may actually be expected, taking into account the actual limitation in surface engineering (nanolithography, etc.). In the following, we will restrict our discussion to the case of flat interfaces, i.e., the pressure difference $\Delta P$ obeys the (nonrestrictive) condition in Eq. (25). As we noted above, a geometry of holes is to be avoided to reduce these effects. This is, however, not actually limiting, as this geometry is not the one that favors large effective slip lengths (see Sec. II C).

Let us consider a practical surface. Of course from the different previous sections, minimizing the friction and maximizing the slip length would require the use of large periodicity $L$. However the latter is limited by stability considerations: the transition to the impregnated Wenzel state, where the liquid fills the roughness and therefore sweeps out 
the gas lubricating pockets, is indeed associated with the disappearance of superlubricating properties. ${ }^{6,19}$ A reasonable compromise may lie in the range $L \sim$ few $\mu \mathrm{m}$ for which pressure differences of tens of mbars-typical for gravitydriven microfluidic flows - can be withstood by an air/water interface. Note that for a precise value of the stability limit, one should actually take into account all surface geometric parameters, including, for instance, the structure height. ${ }^{25}$

Now for microfabricated or nanofabricated surface patterns, the exact geometry of the surface is known a priori and Eqs. (4), (8), (12), and (19) allow us to estimate the expected frictional properties of this surfaces. However, for most superhydrophobic surfaces reported, the structure geometry is not as controlled, and a key parameter such as the solid fraction $\phi_{s}$ has to be estimated or measured. It is therefore very convenient to relate this parameter to a macroscopic observable such as the effective contact angle $\theta_{\text {eff }}$ on the surface. With $\theta_{0}$ the contact angle on the bare surface (in the range of $100-120^{\circ}$ for hydro- or fluorocarbon coatings), $\theta_{\text {eff }}$ relates to $\phi_{s}$ through the Cassie-Baxter relation, ${ }^{5}$

$$
\cos \theta_{\text {eff }}=\phi_{s}\left(\cos \theta_{0}+1\right)-1 .
$$

Fixing the typical height of the structures to $e=L=5 \mu \mathrm{m}$ and assuming a pillars-like type of geometry, it is therefore possible to predict the frictional properties $b_{\text {eff }}$ of the surface as a function of its macroscopic effective contact angle $\theta_{\text {eff }}$. For pillars, Eq. (4) gives

$$
b_{\mathrm{eff}} \simeq \alpha L \sqrt{\frac{\cos \theta_{0}+1}{\cos \theta_{\mathrm{eff}}+1}}-\beta,
$$

with $\alpha=0.325$ and $\beta=0.44$ the numerical factors. This expression shows that close to $\theta_{\text {eff }}=180^{\circ}, b_{\text {eff }}$ strongly diverges, like

$$
b_{\text {eff }} \propto \frac{L}{180-\theta_{\text {eff }}} .
$$

This is apparent in Fig. 8, where huge slippage in the hundreds of micrometers range can be achieved for large yet achievable ${ }^{10}$ contact angles.

It should be noted that for such low solid fractions, the dissipation within the gas sublayer, though small, may have a non negligible effect as it is predicted to decrease the slip length from 110 (solid line) to $60 \mu \mathrm{m}$ (dashed line) for $\theta_{\text {eff }}$ $=179^{\circ}$. As a consequence, the effective slip length at the surface will depend on the liquid viscosity. Let us stress, however, that this dependency is much weaker than what would be predicted by the simple "gas cushion" model, for which $b_{\text {eff }}$ scales as $\eta_{l}$. For the previous example, $\theta_{\text {eff }}$ $=179^{\circ}$, doubling the liquid viscosity only modifies $b_{\text {eff }}$ from 60 to $80 \mu \mathrm{m}$. An effect of fluid viscosity on the slip length has been reported in Ref. 17, however with a much larger magnitude than with the above prediction.

We note in addition that the sublayer dissipation has been calculated assuming no slip of the gas on the solid surfaces, while with a mean free path of order $0.1 \mu \mathrm{m}$ we would anticipate a slip length for the gas onto the solid of the same order. We therefore somewhat overestimate the sublayer dissipation together with its influence on $b_{\text {eff }}$.

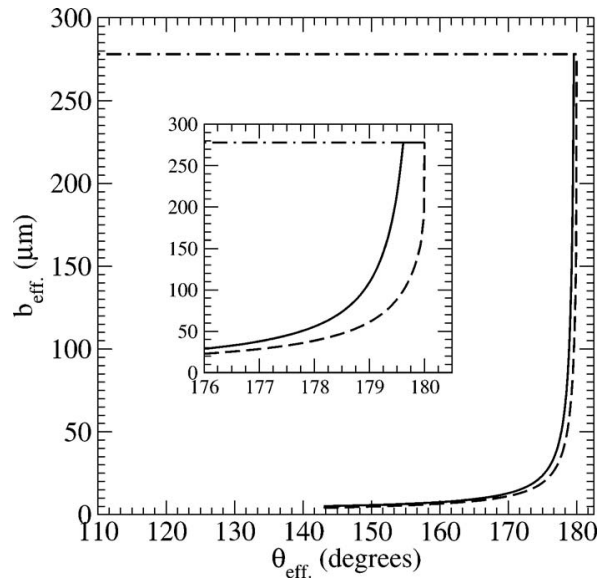

FIG. 8. Effective slip length $b_{\text {eff }}$ as a function of the contact angle $\theta_{\text {eff }}$ on the superhydrophobic surface. The considered surface is made of pillars of height $e=5 \mu \mathrm{m}$ arranged on a square lattice of periodicity $L=5 \mu \mathrm{m}$. The pillars' radius is the only remaining geometrical parameter that controls the overall solid fraction $\phi_{s}$, the latter being related to $\theta_{\text {eff }}$ according to the Cassie relation (26). The viscosity contrast between the liquid and gas corresponds to the water/air interface: $\eta_{l} / \eta_{g}=55.5$. Theoretical expressions are taken according to Table I: (-) theoretical prediction assuming ideal BCs on the composite interface (no-slip and shear-free), (--) theoretical prediction assuming no slip on the solid and a finite dissipation within the gas sublayer (-.-) upper bond of a uniform gas layer $\left(\phi_{s}=0\right)$. Inset: close-up of the near $180^{\circ}$ contact angle region.

Finally, we note that the effect of a finite slip length $b_{s}$ on the solid in the tens of nanometer range does decrease the overall surface friction but still not in large proportion for contact angles below $179^{\circ}$. In the considered example, and for $\theta_{\text {eff }}=179^{\circ}$, it would change the overall slip length from 60 to $65 \mu \mathrm{m}$ (not plotted in Fig. 8). Such an effect is more effective as the effective contact angle $\theta_{\text {eff }}$ is very close to $180^{\circ}$.

In conclusion, we presented here a scaling law analysis that allows us to calculate the frictional properties of superhydrophobic surfaces as a function of the generic geometrical parameters characterizing the surface structure. These laws were successfully tested against numerical calculations for generic geometries and showed that very large slip lengths can be obtained for an ultrahydrophobic surface, characterized by a contact angle very close to $180^{\circ}\left(\theta_{\text {eff }}\right.$ $>178^{\circ}$ ).

Such surfaces may be obtained by present techniques of nano-engineering. For example, a surface made of pillars of $\sim 50-100 \mathrm{~nm}$ in diameter, $5 \mu \mathrm{m}$ apart, is now achievable and corresponds to an effective contact angle $\theta_{\text {eff }}$ larger than $179^{\circ}$. For such surfaces, our analysis predicts slip lengths that can reach hundred of micrometers. Beyond, the slip length is ultimately limited by the dissipation in the gas phase. We hope that our predictions, and the simple analytical laws we provided will motivate further experimental work to develop superlubricating surfaces, with ultralow liquid friction.

\section{ACKNOWLEDGMENTS}

We acknowledge support from ANR PNANO. We thank Elisabeth Charlaix and Jean-Louis Barrat for many discussions on the subject. 
${ }^{1}$ J. Bico, U. Thiele, and D. Quere, "Wetting of textured surfaces," Colloids Surf., A 206, 41 (2002).

${ }^{2}$ A. Lafuma and D. Quere, "Superhydrophobic states," Nature Mater. 2, 457 (2003).

${ }^{3}$ L. C. Gao and T. J. McCarthy, "A commercially available perfectly hydrophobic material (theta(a)/theta(r) $=180$ degrees $/ 180$ degrees)," Langmuir 23, 9125 (2007).

${ }^{4}$ R. N. Wenzel, "Resistance of solid surfaces to wetting by water," Ind. Eng. Chem. 28, 988 (1936).

${ }^{5}$ A. Cassie and S. Baxter, "Wettability of porous surfaces," Trans. Faraday Soc. 40, 546 (1944).

${ }^{6}$ C. Cottin-Bizonne, J. L. Barrat, L. Bocquet, and E. Charlaix, "Lowfriction flows of liquid at nanopatterned interfaces," Nature Mater. 2, 237 (2003).

${ }^{7}$ D. Quere, A. Lafuma, and J. Bico, "Slippy and sticky microtextured solids," Nanotechnology 14, 1109 (2003).

${ }^{8}$ J. Philip, "Flows satisfying mixed no-slip and no-shear conditions," $\mathrm{Z}$. Angew. Math. Phys. 23, 353 (1972).

${ }^{9}$ J. Philip, "Integral properties of flows satisfying mixed no-slip and noshear conditions," Z. Angew. Math. Phys. 23, 960 (1972).

${ }^{10}$ J. W. Kim and C. J. Kim, "Nanostructured surfaces for dramatic reduction of flow resistance in droplet-based microfluidics," Fifteenth IEEE International Conference on Micro Electro Mechanical Systems, Technical Digest (IEEE, Piscataway, NJ, 2002), pp. 479-482.

${ }^{11}$ C. Navier, Mémoire sur les Lois du Louvement des Fluides (l'Académie Royale des Sciences de l'Institut de France, Paris, 1823), Vol. VI.

${ }^{12}$ D. Richard and D. Quere, "Viscous drops rolling on a tilted non-wettable solid," Europhys. Lett. 48, 286 (1999).

${ }^{13}$ J. Ou, B. Perot, and J. P. Rothstein, "Laminar drag reduction in microchannels using ultrahydrophobic surfaces," Phys. Fluids 16, 4635 (2004).

${ }^{14} \mathrm{~J}$. Ou and J. P. Rothstein, "Direct velocity measurements of the flow past drag-reducing ultrahydrophobic surfaces," Phys. Fluids 17, 103606 (2005).

${ }^{15}$ S. Gogte, P. Vorobieff, R. Truesdell, A. Mammoli, F. van Swol, P. Shah, and C. J. Brinker, "Effective slip on textured superhydrophobic surfaces," Phys. Fluids 17, 051701 (2005).

${ }^{16}$ C. H. Choi, U. Ulmanella, J. Kim, C. M. Ho, and C. J. Kim, "Effective slip and friction reduction in nanograted superhydrophobic microchannels," Phys. Fluids 18, 087105 (2006).

${ }^{17}$ C. H. Choi and C. J. Kim, "Large slip of aqueous liquid flow over a nanoengineered superhydrophobic surface," Phys. Rev. Lett. 96, 066001 (2006).

${ }^{18}$ L. Bocquet, P. Tabeling, and S. Manneville, "Comment on large slip of aqueous liquid flow over a nanoengineered superhydrophobic surface," Phys. Rev. Lett. 97, 109601 (2006).

${ }^{19}$ P. Joseph, C. Cottin-Bizonne, J. M. Benoit, C. Ybert, C. Journet, P. Tabeling, and L. Bocquet, "Slippage of water past superhydrophobic carbon nanotube forests in microchannels," Phys. Rev. Lett. 97, 156104 (2006).

${ }^{20}$ O. I. Vinogradova and G. E. Yakubov, "Dynamic effects on force mea- surements. 2. Lubrication and the atomic force microscope," Langmuir 19, 1227 (2003).

${ }^{21}$ C. Cottin-Bizonne, B. Cross, A. Steinberger, and E. Charlaix, "Boundary slip on smooth hydrophobic surfaces: Intrinsic effects and possible artifacts," Phys. Rev. Lett. 94, 056102 (2005).

${ }^{22}$ L. Joly, C. Ybert, and L. Bocquet, "Probing the nanohydrodynamics at liquid-solid interfaces using thermal motion," Phys. Rev. Lett. 96, 046101 (2006).

${ }^{23}$ P. Huang and K. S. Breuer, "Direct measurement of slip length in electrolyte solutions," Phys. Fluids 19, 028104 (2007).

${ }^{24}$ E. Lauga and H. A. Stone, "Effective slip in pressure-driven Stokes flow," J. Fluid Mech. 489, 55 (2003).

${ }^{25}$ C. Cottin-Bizonne, C. Barentin, E. Charlaix, L. Bocquet, and J. L. Barrat, "Dynamics of simple liquids at heterogeneous surfaces: Moleculardynamics simulations and hydrodynamic description," Eur. Phys. J. E 15, 427 (2004).

${ }^{26}$ M. Sbragaglia, R. Benzi, L. Biferale, S. Succi, and F. Toschi, "Surface roughness-hydrophobicity coupling in microchannel and nanochannel flows," Phys. Rev. Lett. 97, 204503 (2006).

${ }^{27}$ S. Richardson, "On the no-slip boundary-condition," J. Fluid Mech. 59, 707 (1973)

${ }^{28}$ E. Lauga, M. P. Brenner, and H. A. Stone, Handbook of Experimental Fluid Dynamics (Springer, New York, 2005).

${ }^{29}$ O. I. Vinogradova, "Drainage of a thin liquid-film confined between hydrophobic surfaces," Langmuir 11, 2213 (1995).

${ }^{30}$ P. de Gennes, "On fluid wall slippage," Langmuir 18, 3413 (2002).

${ }^{31}$ C. Y. Wang, "Flow over a surface with parallel grooves," Phys. Fluids 15, 1114 (2003)

${ }^{32} \mathrm{H}$. Brinkman, "On the permeability of media consisting of closely packed porous particles," Appl. Sci. Res., Sect. A 1, 81 (1947).

${ }^{33}$ A. S. Sangani and A. Acrivos, "Slow flow through a periodic array of spheres," Int. J. Multiphase Flow 8, 343 (1982).

${ }^{34}$ G. S. Beavers and D. D. Joseph, "Boundary conditions at naturally permeable wall," J. Fluid Mech. 30, 197 (1967).

${ }^{35}$ A. Steinberger, C. Cottin-Bizonne, P. Kleimann, and E. Charlaix, "High friction on a bubble mattress," Nature Mater. 6, 665 (2007).

${ }^{36}$ M. Sbragaglia and A. Prosperetti, "A note on the effective slip properties for microchannel flows with ultra-hydrophobic surfaces," Phys. Fluids 19, 043603 (2007)

${ }^{37}$ P. Panzer, M. Liu, and D. Einzel, "The effects of boundary curvature on hydrodynamic fluid-flow: Calculation of slip lengths," Int. J. Mod. Phys. B 6, 3251 (1992).

${ }^{38} \mathrm{~W}$. Rybczyński, "On the translatory motion of a fluid sphere in a viscous medium (in german)," Bull. Int. Acad. Pol. Sci. Lett., Cl. Sci. Math. Nat., Ser. A 2, 40 (1911).

${ }^{39}$ J. Hadamard, "Mouvement permanent lent d'une sphère liquide et visqueuse dans un liquide visqueux," C. R. Acad. Sci. Paris 152, 1735 (1911). 